\title{
A comparison of clinical performance between i-gel and endotracheal tube in pediatric laparoscopic surgeries
}

Received August 20, 2018

Revised 1st, September 27, 2018

2nd, October 10, 2018

Accepted October 11, 2018

\section{Corresponding author}

Jeong Ho Kim, M.D.

Department of Anesthesiology and Pain Medicine, Dong-A

University School of Medicine, 32

Daesingongwon-ro, Seo-gu, Busan

49201, Korea

Tel: 82-51-240-5390

Fax: 82-51-247-7819

E-mail: surfy07@gmail.com

\section{ORCID}

https://orcid.org/0000-0003-4447-2838

\section{Jeong In Hong, Tae Young Lee, Sang Yoong Park, So Ron Choi, Seung-Cheol Lee, Chan Jong Chung, and Jeong Ho Kim}

Department of Anesthesiology and Pain Medicine, Dong-A University School of Medicine, Busan, Korea

\begin{abstract}
Background: The current evidence on the safe use of supraglottic airway for pediatric laparoscopic surgeries is limited. Although i-gel has been successfully used in adult laparoscopic surgeries, to our knowledge, no studies have compared it to the endotracheal tube (ETT) in pediatric laparoscopic surgeries. This study evaluated the effectiveness of i-gel over ETT with regards to the respiratory and hemodynamic parameters during pediatric laparoscopic surgeries.
\end{abstract}

Methods: A total of 60 pediatric patients undergoing elective laparoscopic surgeries were randomly allocated to either the i-gel or ETT groups. Anesthetics used included ketamine, sevoflurane, and rocuronium. The primary outcome measured was the peak airway pressure (PAP) and the secondary outcomes measured were leak fraction, endtidal $\mathrm{CO}_{2}$, respiratory rate, insertion time, heart rate, blood pressure and perioperative complications.

Results: The PAP during surgeries was higher in the ETT group than in the i-gel group. There were no statistically significant differences in the leak fraction, end-tidal $\mathrm{CO}_{2}$, and respiratory rate. The i-gel group had a shorter insertion time compared with the ETT group. The changes in heart rate were comparable in both groups. However, systolic and diastolic pressures were higher in the ETT group following intubation, before and after the creation of pneumoperitoneum. The incidence of perioperative complications was similar in both groups.

Conclusions: The i-gel provided adequate ventilation with lower PAP compared with ETT. In addition, it provided minimal hemodynamic changes compared with ETT. Therefore, the i-gel may provide a suitable alternative to ETT in pediatric laparoscopic surgeries.

Keywords: Intubation, intratracheal; I-gel; Laparoscopy; Pediatrics.

\section{INTRODUCTION}

Laparoscopy has extended to several procedures including herniorrhaphy, high ligation, appendectomy, and advanced gastrointestinal procedures. It has several advantages over conventional surgery. As laparoscopy is less invasive, it is associated with reduced postoperative pain and a short hospital stay [1]. For this reason, laparoscopic surgery has gained an increasing popularity among the pediatric population. However, because children exhibit lower functional residual capacity than adults, they are more likely to be affected by laparoscopic surgery and may have difficulty in maintaining

This is an Open Access article distributed under the terms of the Creative Commons Attribution Non-Commercial License (http://creativecommons.org/licenses/by-nc/4.0) which permits unrestricted non-commercial use, distribution, and reproduction in any medium, provided the original work is properly cited.

Copyright (c) the Korean Society of Anesthesiologists, 2019 
airway patency, compared with adults $[2,3]$. Therefore, the elevation of intra-abdominal pressure during anesthesia may be challenging in pediatric laparoscopic surgeries.

The cuffed endotracheal tube has been considered the gold standard for laparoscopic surgeries. Since the introduction of supraglottic airway devices (SADs), several studies comparing laryngeal mask airway (LMA) to endotracheal tube (ETT) have been conducted, with a few describing the clinical effectiveness of LMA in pediatric laparoscopic surgeries $[4,5]$.

Recently, several-second generation SADs have been used in laparoscopic surgeries. The i-gel (i-gel ${ }^{\mathrm{TM}}$, Intersurgical Ltd., UK) is a disposable second-generation SAD made of a medical-grade thermoplastic elastomer. It has a non-inflatable cuff to reduce complications associated with compression trauma and high pressure [6]. Moreover, the insertion of igel is easier than other SADs, and i-gel is capable of sealing higher oropharyngeal leak pressure (OLP), compared with first-generation SADs [7-9]. As a result of these advantages, i-gel has been successfully used in adult laparoscopic surgeries [10-12]. However, to our knowledge, no previous studies have compared i-gel with ETT in pediatric laparoscopic surgeries.

This prospective randomized study investigated respiratory parameters, as well as perioperative hemodynamic changes and complications associated with the use of i-gel and ETT in pediatric patients undergoing laparoscopic surgery under general anesthesia. The primary outcome measured was the peak airway pressure (PAP); the secondary outcomes measured were leak fraction, end-tidal $\mathrm{CO}_{2}$, respiratory rate, insertion time, heart rate, blood pressure and perioperative complications. We predicted that i-gel would provide adequate ventilation, minimal hemodynamic change, and fewer complications, compared with ETT.

\section{MATERIALS AND METHODS}

This study was approved by Institutional Research Board of our University Hospital (IRB-17-030). After we obtained their parents' written informed consents, 60 pediatric patients under 10 years old, with an American Society of Anesthesiologists physical status I or II, who were undergoing elective laparoscopic surgeries in the supine position, were randomly allocated to either the i-gel or ETT groups. Allocation concealment was performed using the sealed envelope method.
Patients with upper respiratory infection, pathology of the neck or upper respiratory tract, potential difficult intubation, or an increased risk of aspiration were excluded. In addition, patients with morbid obesity were excluded from the study due to its influence on airway quality and lung compliance.

All elective laparoscopic surgeries were performed under standard general anesthesia. Patients fasted for $6 \mathrm{~h}$ prior to the surgery. They were premedicated with intramuscular glycopyrrolate $0.004 \mathrm{mg} / \mathrm{kg}$, $30 \mathrm{~min}$ prior to induction. In the operating room, non-invasive blood pressure, pulse oximetry, and electrocardiography were used for monitoring. Anesthesia was induced using intravenous ketamine $2 \mathrm{mg} /$ $\mathrm{kg}$ and rocuronium $0.6 \mathrm{mg} / \mathrm{kg}$. Manual bag-mask ventilation was maintained for $3 \mathrm{~min}$ before device insertion. Neuromuscular blockade was monitored by the train-of-four (TOF) stimulator (EZStim II, Life-Tech Inc., USA) throughout the surgeries. Device insertion was performed after confirming a TOF count of zero.

In the ETT group, cuffed tubes (Covidien, USA) were used. Appropriate ETT sizes were selected using modified Cole's formula [13]. Infants under the age of 1 year had $3.0 \mathrm{~mm}$ or 3.5 $\mathrm{mm}$ tubes at the discretion of the investigator. The ETT was exchanged with the next smaller size ( $0.5 \mathrm{~mm}$ smaller) when resistance with intubation was faced; in cases of leakage, the cuff was inflated with minimal cuff pressure required to seal.

In the i-gel group, the size was selected according to the manufacturer's instructions and depending on the body weight [6]. Proper positioning of the i-gel was confirmed when bilateral clear lung sounds with no audible leaks were heard on auscultation, square-wave capnography, and adequate chest rise on manual ventilation. When i-gel failed to maintain sufficient ventilation, proper manipulation (modulation of the device or changing the neck position) was performed. If ventilation was insufficient despite proper manipulation, the device was removed and replaced with an appropriate ETT. We excluded cases that involved a change in ETT size, replacement of the i-gel with ETT, or conversion to open surgery, due to potential bias in the study results.

All procedures were performed by a single anesthesiologist with experience performing nearly 100 insertions using each of the devices in pediatric patients. In addition, all operations were performed by the same surgeon. The hemodynamic parameters were recorded by a blinded observer, who monitored heart rate and blood pressure outside of the operating 
room. However, an unblinded observer collected the respiratory data.

Following the insertion of devices, to measure the OLP, the expiratory valve of the circle system was closed at a fresh gas flow of $3 \mathrm{~L} / \mathrm{min}$ until an audible gas leakage was heard over neck by auscultation with a stethoscope [14]. The OLP was not allowed to exceed $30 \mathrm{cmH}_{2} \mathrm{O}$ to avoid barotrauma. After measurement of the OLP, volume-controlled ventilation was initiated using a tidal volume of $8 \mathrm{ml} / \mathrm{kg}$. Monitoring of mechanical ventilation and respiratory parameters was performed by using a Primus Dräger anesthesia ventilator (Dräger Medical GmbH, Germany). Anesthesia was maintained with $2-3 \%$ sevoflurane and $40 \%$ oxygen in air, with a fresh gas flow rate of $3 \mathrm{~L} / \mathrm{min}$. Insertion time was defined as time from removal of the face mask to the first capnography trace. Respiratory rate was controlled to maintain end-tidal $\mathrm{CO}_{2}$ between 35-45 $\mathrm{mmHg}$ with an inspiratory-to-expiratory ratio of 1:2. Pneumoperitoneum was maintained at 8-10 mmHg. During pneumoperitoneum, minute ventilation was increased by adjusting the respiratory rate.

PAP, leak fraction, end-tidal $\mathrm{CO}_{2}$, respiratory rate, heart rate, and blood pressure were recorded after intubation (T1), 5 min before (T2) and after (T3) the creation of pneumoperitoneum, and $5 \mathrm{~min}$ before (T4) and after (T5) the end of pneumoperitoneum. Basal heart rate and blood pressure were recorded before intubation (T0).

Insufficient ventilation was defined as an expiratory tidal volume lower than $5 \mathrm{ml} / \mathrm{kg}$. The difference between the inspired and expired tidal volumes was used to calculate the leak volume. The leak fraction was defined as the leak volume divided by the inspired tidal volume and expressed as a percentage. The quality of ventilation was classified into four categories according to the lung sounds heard on auscultation: clear, minimal obstruction, partial obstruction, and complete obstruction [15]; it was assessed at the onset of adequate ventilation, before and after pneumoperitoneum, and when respiratory parameters were abnormal. During anesthetic management, any changes in the quality of ventilation and manipulation were recorded.

At the end of surgery, sevoflurane was stopped and paralysis was reversed using pyridostigmine and glycopyrrolate when the TOF count was 4 . Before discharge from the postanesthetic care unit, blood on device, desaturation $\left(\mathrm{SpO}_{2}<\right.$ $90 \%)$, cough, laryngospasm, stridor, regurgitation, aspiration, and vomiting were recorded.

The sample size was based on data from a pilot study of 20 patients in the i-gel and ETT groups (10 patients each), in which the baseline PAP measurements were $13.5 \pm 2.0$ $\mathrm{cmH}_{2} \mathrm{O}$ and $15.3 \pm 2.4 \mathrm{cmH}_{2} \mathrm{O}$, respectively. A sample size calculation using data with a two-sided alpha of 0.05 and $80 \%$ power determined that 25 subjects were needed to detect a statistically significant difference between groups. The study enrolled 60 patients (30 in each group) to allow for potential dropout.

The data were analyzed using the SPSS software (version 23.0, IBM Co., USA). The results are expressed as the median (interquartile range, IQR) or number. We tested continuous data for normal distribution using the Kolmogorov-Smirnov

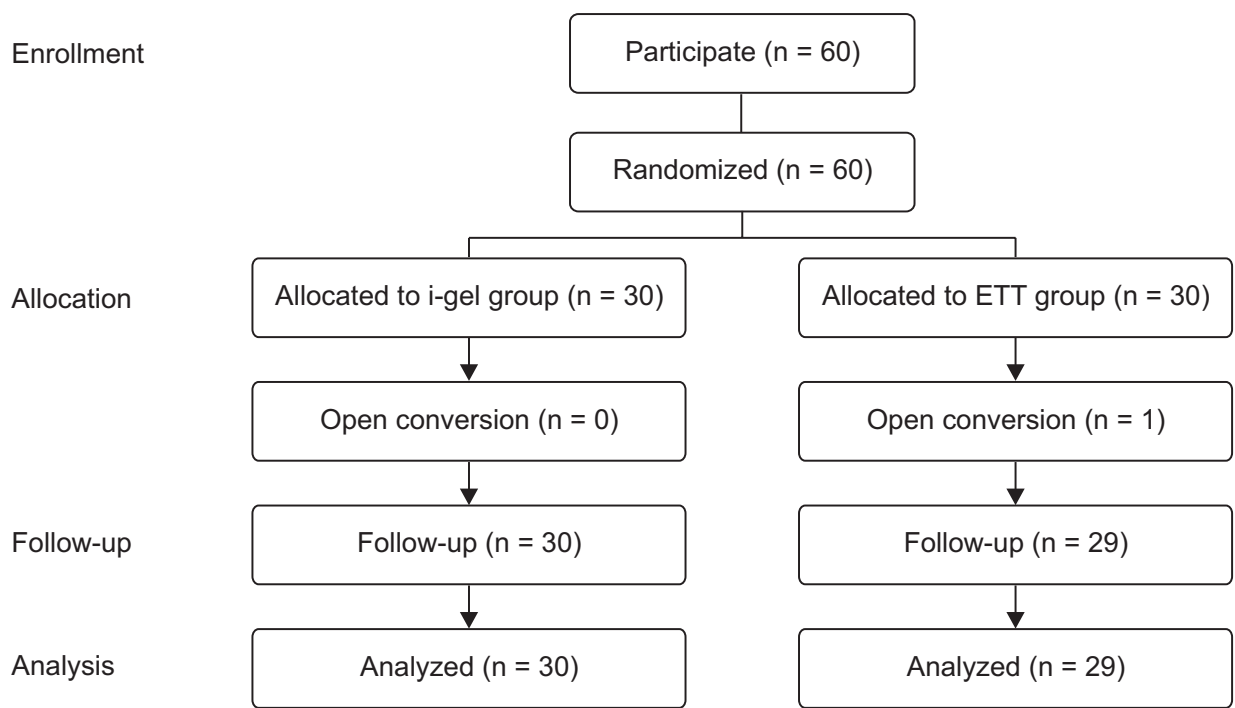

Fig. 1. Consort flow diagram. ETT: endotracheal tube. 
test. The continuous data (age, height, weight, pneumoperitoneum time, anesthetic time, peak airway pressure, leak fraction, end-tidal $\mathrm{CO}_{2}$, respiratory rate, insertion time, heart rate, and blood pressure) were analyzed with Mann-Whitney tests. The categorical data (sex, operative type, insertion attempts, quality of ventilation, and perioperative complications) were analyzed using the chi-squared test or Fisher's exact test. A value of $\mathrm{P}<0.05$ was considered statistically significant.

\section{RESULTS}

In total, 60 patients were enrolled in the study; 30 patients each were assigned to the i-gel and ETT groups. In the ETT group, one patient was excluded because laparoscopy was converted to laparotomy (Fig. 1). There were no significant differences in the demographic data, types of surgeries performed, or durations of pneumoperitoneum and anesthetic times between the two groups (Table 1). Median (IQR) OLP of the i-gel was $25.0(22.0,27.0) \mathrm{cmH}_{2} \mathrm{O}$.

The PAP during surgeries was consistently higher in the ETT group than in the i-gel group $(\mathrm{P}<0.001$ at all time points). The leak fraction was similar in both groups (Fig. 2). Moreover, end-tidal $\mathrm{CO}_{2}$ and respiratory rate showed no differences between both groups (Fig. 3). The i-gel group had a shorter insertion time, compared with the ETT group $(\mathrm{P}<$ 0.001) (Table 2). Changes in heart rate during surgeries were similar in both groups. However, systolic $(\mathrm{P}=0.001,0.002$, and 0.001 respectively) and diastolic $(\mathrm{P}<0.001, \mathrm{P}=0.003$, and
$\mathrm{P}<0.001$ respectively) blood pressures differed between the groups following intubation, as well as before and after the creation of pneumoperitoneum (Fig. 4).

Minor perioperative complications occurred in a few cases in both groups; however, their incidences were similar ( $\mathrm{P}$ $=0.080$, Table 3 ). During the surgery, no displacement of igel occurred and ventilation was adequately maintained. In both groups, all patients exhibited clear quality of ventilation throughout the surgery.

All patients in both groups were successfully intubated at the initial trial. Most of the patients in the i-gel group were adequately ventilated without any manipulation; however,

Table 1. Patient Characteristics and Operation Data

\begin{tabular}{lccc}
\hline & i-gel $(\mathrm{n}=30)$ & $\mathrm{ETT}(\mathrm{n}=29)$ & P value \\
\hline Age $(\mathrm{mo})$ & $26.5(12.0,48.0)$ & $25.0(14.0,52.5)$ & 0.885 \\
Sex $(\mathrm{M} / \mathrm{F})$ & $16 / 14$ & $19 / 10$ & 0.430 \\
Height $(\mathrm{cm})$ & $96.0(75.0,107.0)$ & $90.0(77.0,107.2)$ & 0.897 \\
Weight $(\mathrm{kg})$ & $13.5(9.1,19.0)$ & $13.0(11.0,19.5)$ & 0.808 \\
Operative type & & & 0.874 \\
$\quad$ High ligation & 19 & 18 & \\
$\quad$ Herniorrhaphy & 9 & 8 & \\
$\quad$ Others* & 2 & 3 & \\
Pneumoperitoneum & $40.0(28.7,51.2)$ & $40.0(30.0,45.0)$ & 0.459 \\
$\quad$ time (min) & & & \\
Anesthetic & $65.0(53.7,76.2)$ & $65.0(52.5,75.0)$ & 0.893 \\
$\quad$ time (min) & & & \\
\hline
\end{tabular}

Values are presented as median (1Q, 3Q) or number only. ETT: endotracheal tube. *Others include laparoscopic appendectomy $(n=2)$ in the I-gel group, pyloromyotomy $(n=2)$ and omentectomy $(n=1)$ in the ETT group.
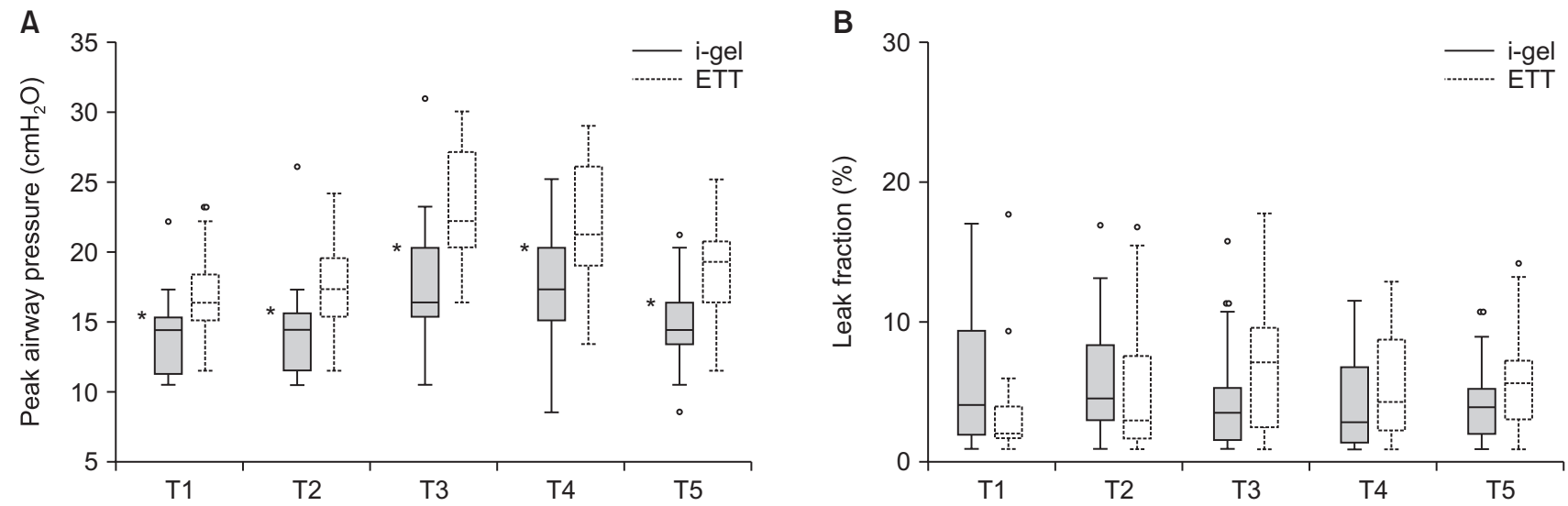

Fig. 2. Box plot graphic of the peak airway pressure (A) and the leak fraction (B). Values are presented as median (1Q, 3Q). ETT: endotracheal tube, T1: after intubation, T2 and T3: 5 min before and after the beginning of pneumoperitoneum, respectively, T4 and T5: 5 min before and after the end of pneumoperitoneum, respectively. ${ }^{*} \mathrm{P}<0.05$ compared to the ETT group. 
A

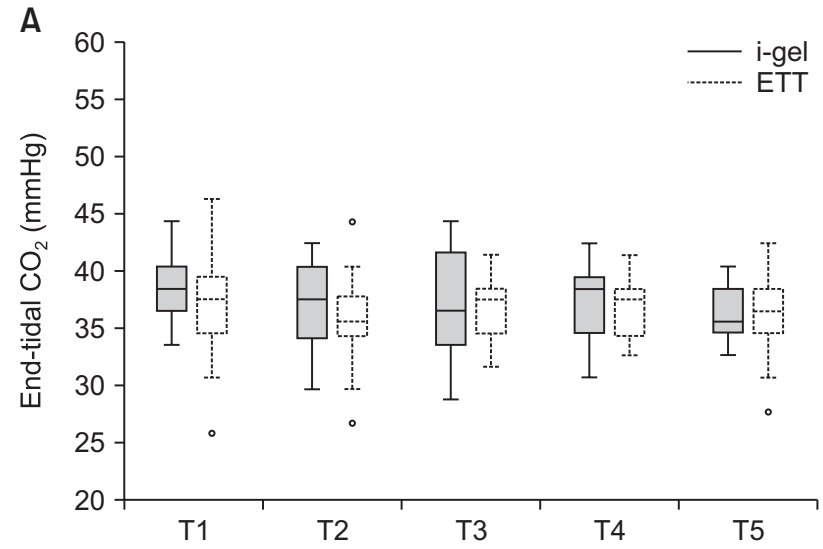

B

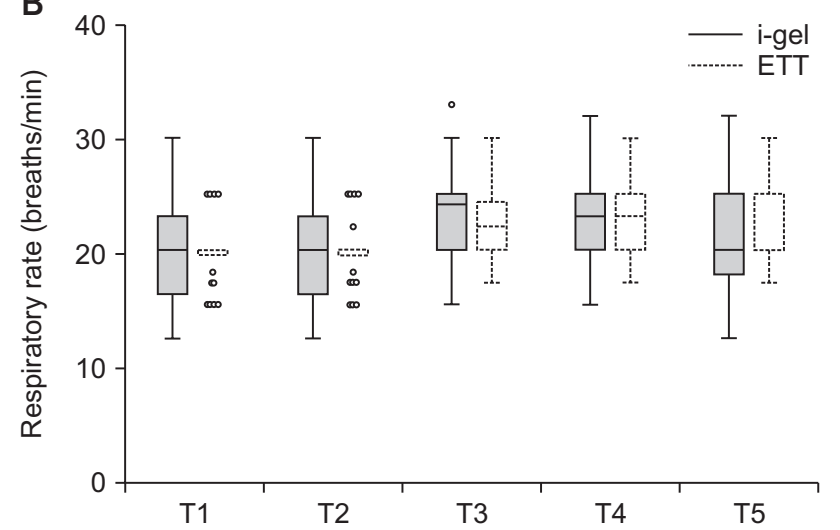

Fig. 3. Box plot graphic of the end-tidal $\mathrm{CO}_{2}(\mathrm{~A})$ and respiratory rate (B). Values are presented as median (1Q, 3Q). ETT: endotracheal tube, $\mathrm{T} 1$ : after intubation, T2 and T3: 5 min before and after the beginning of pneumoperitoneum, respectively, T4 and T5: 5 min before and after the end of pneumoperitoneum, respectively.

Table 2. Comparative Data for the i-gel and the ETT

\begin{tabular}{lccr}
\hline & i-gel $(\mathrm{n}=30)$ & ETT $(\mathrm{n}=29)$ & P value \\
\hline Size $(\mathrm{mm})$ & & & \\
1.0/1.5/2.0/2.5 & $2 / 9 / 15 / 4$ & & \\
3.0/3.5/4.0/4.5/5.0/5.5 & & $1 / 1 / 13 / 5 / 8 / 1$ & \\
Insertion attempts (1/2) & $30 / 0$ & $29 / 0$ & 1.000 \\
Insertion time (s) & 18.0 & 32.0 & $<0.001$ \\
& $(16.8,20.0)$ & $(30.0,35.0)$ & \\
Quality of ventilation* & $30 / 0 / 0 / 0$ & $29 / 0 / 0 / 0$ & 1.000
\end{tabular}

Values are presented as number only or median (1Q, 3Q). ETT: endotracheal tube. *Quality of ventilation is assorted into clear/minimal obstruction/partial obstruction/complete obstruction.

three patients experienced a dislodged insertion, which was corrected by simple manipulation, such as pulling or pushing the device and changing the neck position.

\section{DISCUSSION}

Laparoscopic surgery is associated with increased abdominal pressure, which increases PAP and decreases lung compliance. Importantly, lung compliance is defined as a change in lung volume divided by change in airway pressure; reduced compliance impedes adequate pulmonary gas exchange and predisposes a patient to hypercarbia [2]. Bannister et al. [3] reported significant changes in pulmonary mechanics during laparoscopic surgical procedures in infants. In the present study, ETT group consistently showed a higher PAP compared with the i-gel group. Lai et al. [12] proposed that this was due to the larger internal diameter of i-gel, compared with that of ETT. In addition, ETT produces reflex bronchoconstriction more than laryngeal airway mask, which may lead to differences in PAP. Because of these characteristics of laryngeal mask airway, Berry et al. [16] reported that laryngeal mask airway is effective for the maintainance of intraoperative pulmonary function and reduction of the risk for atelectasis and pulmonary infection. Thus, i-gel seems to have several advantages over ETT, with respect to airway management in pediatric laparoscopic surgeries.

The difference between the OLP and the PAP is considered the most important factor when evaluating the adequacy of SADs for laparoscopic procedures. An SAD with a high OLP is recommended for high inspiratory pressure and reduced lung compliance during pediatric laparoscopic surgeries [2]. In the present study, the median OLP of the i-gel group was higher than the OLP in other studies $\left(20 \mathrm{cmH}_{2} \mathrm{O}\right.$, respectively) $[17,18]$. i-gel provides ventilation with low PAP and high OLP; thus it is suspected to exhibit a wide safety margin and effective sealing for pediatric laparoscopic surgery.

Unlike other SADs, i-gel does not have an inflatable cuff and was theoretically presumed to have high leak fraction. However, data from this study show that leak fraction during surgeries was not significantly different between the two groups, which suggests that the i-gel is equally effective in providing adequate ventilation during laparoscopic surgery. In addition, end-tidal $\mathrm{CO}_{2}$ and respiration rate in both groups did not significantly differ, which is consistent with previous studies $[4,5,10]$.

The mean insertion time of i-gel was significantly shorter than that of ETT. In other studies, i-gel was reported to have a shorter insertion time than ETT and other SADs $[9,11,19]$. We 

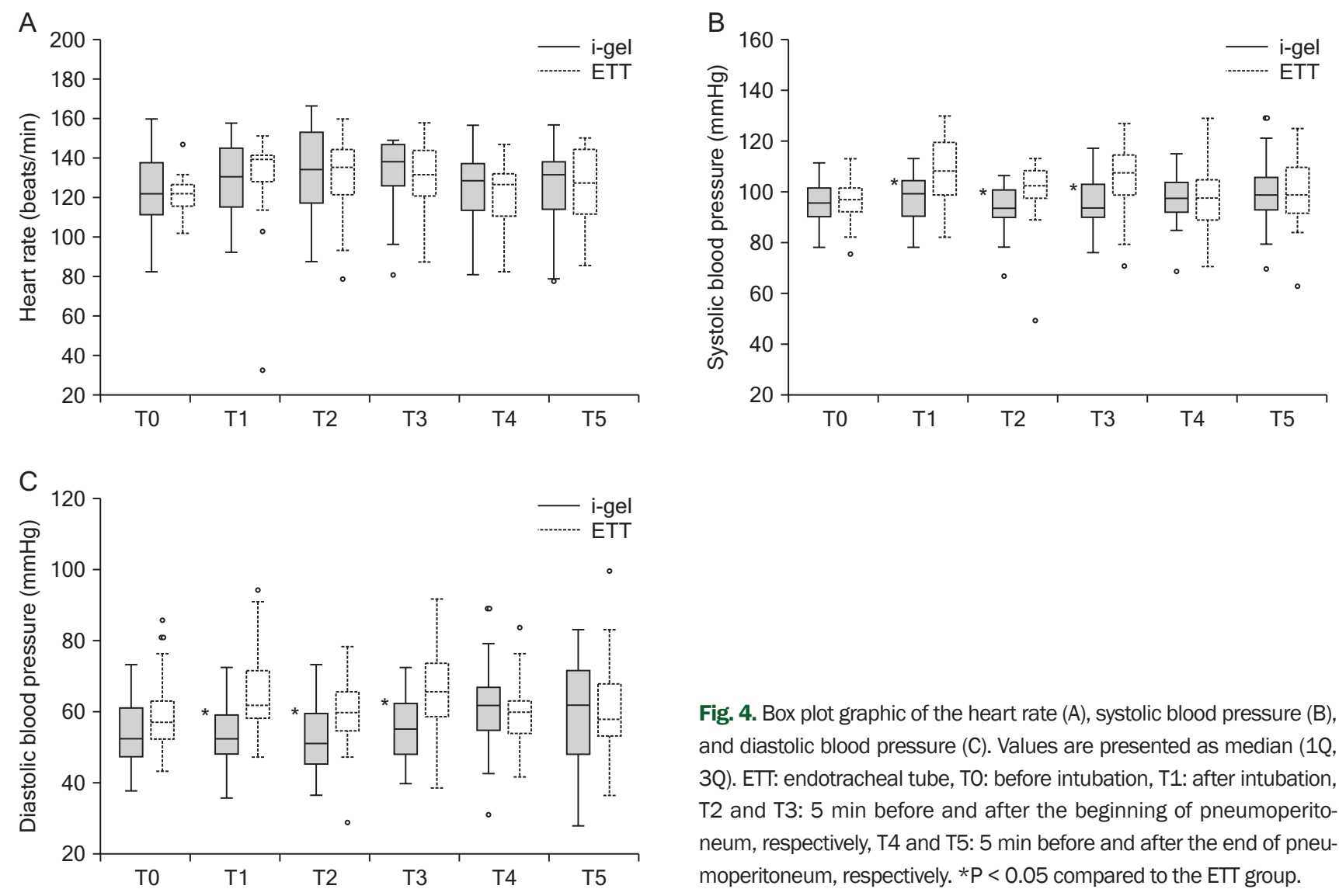

Fig. 4. Box plot graphic of the heart rate (A), systolic blood pressure (B), and diastolic blood pressure $(\mathrm{C})$. Values are presented as median (1Q, 3Q). ETT: endotracheal tube, TO: before intubation, T1: after intubation, T2 and T3: 5 min before and after the beginning of pneumoperitoneum, respectively, T4 and T5: 5 min before and after the end of pneumoperitoneum, respectively. ${ }^{*} \mathrm{P}<0.05$ compared to the ETT group.

Table 3. Perioperative Complications

\begin{tabular}{lcc}
\hline & i-gel $(\mathrm{n}=30)$ & ETT $(\mathrm{n}=29)$ \\
\hline None & 28 & 22 \\
Severe air leakage* $^{*}$ & 0 & 0 \\
Airway obstruction & 0 & 0 \\
Desaturation $\left(\mathrm{SpO}_{2}<90 \%\right)$ & 0 & 2 \\
Blood on device & 1 & 0 \\
Cough & 1 & 3 \\
Laryngospasm/stridor & 0 & 0 \\
Regurgitation/aspiration & 0 & 0 \\
Vomiting & 2 & 3 \\
\hline
\end{tabular}

Values are presented as number only. ETT: endotracheal tube. *Expiratory tidal volume less than $5 \mathrm{ml} / \mathrm{kg}$.

suspecte that the need for laryngoscopy-guided intubation in cases of ETT may have increased the insertion time. Hardman and Wills [20] reported that in the absence of preoxygenation, with an open airway in an 8-year-old child, arterial hemoglobin oxygen saturation $\left(\mathrm{SaO}_{2}\right)$ of $90 \%$ was reached in $0.51 \mathrm{~min}$ from the start of apnea. Lower age was associated with a shorter time. Moreover, achievement of effective preoxygenation in the young child is often more difficult. Thus, the difference of insertion time between these two devices is

considered clinically meaningful in children.

Blood pressure was higher in the ETT group, compared with the i-gel group, following intubation, as well as $5 \mathrm{~min}$ before and after the creation of pneumoperitoneum. Oropharyngeal and laryngotracheal stimulation subsequent to laryngoscopy and tracheal intubation provoke sympathoadrenal discharge, leading to hemodynamic changes [21]. Moreover, insufflation of the peritoneal cavity may increase blood pressure by increasing the systemic vascular resistance [2]. Badheka et al. [10] reported that i-gel caused fewer hemodynamic changes, compared with ETT, in adult laparoscopic surgeries. The higher blood pressure in the ETT group even after $5 \mathrm{~min}$ of pneumoperitoneum is most likely due to the short time (in range of 10-15 $\mathrm{min}$ ) to pneumoperitoneum after intubation. The effect of laryngoscopic tracheal intubation could persist until the creation of pneumoperitoneum. However, unlike other studies, changes in heart rate showed similar trends in both groups [10].

We did not observe any regurgitation or aspiration in either group. In addition, the incidence of vomiting was comparable between both groups. In the i-gel group, oxygenation 
was well- maintained with no critical events occurring during surgeries. However, two patients in the ETT group showed instantaneous desaturation before spontaneously recovering. This may have occurred because extubation was performed before muscle relaxation was sufficiently reversed, or as a result of temporary bronchospasm. The incidences of blood on device, cough, laryngospasm, and stridor were not significantly different between the two groups. Overall, there was no significant difference between the groups in the incidence of postoperative complications. The results of this study are comparable with other studies evaluating SADs in adult laparoscopic surgeries $[4,10]$. However, some authors reported that adverse events including cough and sore throat, were more common in the ETT group than in the SAD group $[5,12]$. The non-inflatable cuff of the i-gel provides low pressure on the mucosa, which reduces compression in the microvascular structure and peri-laryngeal tissues [22,23]. Differences in the results of these studies may be related to the proficiency of the device user and the sample size. In addition, complaints of sore throat and dysphagia, easily observed among adults, were difficult to monitor properly in the small children.

The i-gel and ETT insertions were successful on the initial attempt in all patients, although simple manipulation was needed in three patients in the i-gel group to maintain adequate ventilation. In other studies, i-gel showed similar success rates $[11,19,24,25]$.

There were several limitations in this study. Only patients with normal airway anatomy, short operating times, and supine position were included. Therefore, it is difficult to generalize these results to other patients. More studies are required to test the adequacy of i-gel in different groups. Second, an unblinded observer collected respiratory data. Thus, we were unable to ensure that no bias was involved. Third, the number of patients, receiving size 1.0 was relatively small.

In conclusion, the i-gel provided adequate ventilation with lower PAP, and demonstrated a shorter insertion time and minimal hemodynamic changes when compared with ETT. Hence, i-gel can provide a suitable alternative to ETT in pediatric laparoscopic surgeries.

\section{CONFLICTS OF INTEREST}

No potential conflict of interest relevant to this article was reported.

\section{ORCID}

Jeong In Hong: https://orcid.org/0000-0002-9487-3368

Tae Young Lee: https://orcid.org/0000-0002-9241-9735

Sang Yoong Park: https://orcid.org/0000-0001-7495-8025

So Ron Choi: https://orcid.org/0000-0002-4173-8939

Seung-Cheol Lee: https://orcid.org/0000-0001-8669-5517

Chan Jong Chung: https://orcid.org/0000-0002-0236-7135

\section{REFERENCES}

1. Valenza F, Chevallard G, Fossali T, Salice V, Pizzocri M, Gattinoni L. Management of mechanical ventilation during laparoscopic surgery. Best Pract Res Clin Anaesthesiol 2010; 24: 227-41.

2. Manner T, Aantaa R, Alanen M. Lung compliance during laparoscopic surgery in paediatric patients. Paediatr Anaesth 1998; 8: 25-9.

3. Bannister CF, Brosius KK, Wulkan M. The effect of insufflation pressure on pulmonary mechanics in infants during laparoscopic surgical procedures. Paediatr Anaesth 2003; 13: 785-9.

4. Ozdamar D, Güvenç BH, Toker K, Solak M, Ekingen G. Comparison of the effect of LMA and ETT on ventilation and intragastric pressure in pediatric laparoscopic procedures. Minerva Anestesiol 2010; 76: 592-9.

5. Sinha A, Sharma B, Sood J. ProSeal as an alternative to endotracheal intubation in pediatric laparoscopy. Paediatr Anaesth 2007; 17: 327-32.

6. Intersurgical, i-gel user guide [Internet]. Wokingham, Intersurgical Ltd. 2017 [cited 2016 Dec 3]. Available from http://www.i-gel. com.

7. Levitan RM, Kinkle WC. Initial anatomic investigations of the igel airway: a novel supraglottic airway without inflatable cuff. Anaesthesia. 2005; 60: 1022-6.

8. Wharton NM, Gibbison B, Gabbott DA, Haslam GM, Muchatuta $\mathrm{N}$, Cook TM. I-gel insertion by novices in manikins and patients. Anaesthesia 2008; 63: 991-5.

9. Theiler LG, Kleine-Brueggeney M, Luepold B, Stucki F, Seiler S, Urwyler N, et al. Performance of the pediatric-sized i-gel compared with the Ambu AuraOnce laryngeal mask in anesthetized and ventilated children. Anesthesiology 2011; 115: 102-10.

10. Badheka JP, Jadliwala RM, Chhaya VA, Parmar VS, Vasani A, Rajyaguru AM. I-gel as an alternative to endotracheal tube in adult laparoscopic surgeries: a comparative study. J Minim Access Surg 2015; 11: 251-6.

11. Park SY, Rim JC, Kim H, Lee JH, Chung CJ. Comparison of i-gel ${ }^{\circledR}$ and LMA Supreme ${ }^{\circledR}$ during laparoscopic cholecystectomy. Korean J Anesthesiol 2015; 68: 455-61. 
12. Lai CJ, Liu CM, Wu CY, Tsai FF, Tseng PH, Fan SZ. I-Gel is a suitable alternative to endotracheal tubes in the laparoscopic pneumoperitoneum and trendelenburg position. BMC Anesthesiol 2017; 17:3.

13. Cole F. Pediatric formulas for the anesthesiologist. AMA J Dis Child 1957; 94: 672-3.

14. Lopez-Gil M, Brimacombe J, Keller C. A comparison of four methods for assessing oropharyngeal leak pressure with the laryngeal mask airway (LMA) in paediatric patients. Paediatr Anaesth 2001; 11:319-21.

15. Cook TM, Gatward JJ, Handel J, Hardy R, Thompson C, Srivastava R, et al. Evaluation of the LMA Supreme in 100 non-paralysed patients. Anaesthesia 2009; 64: 555-62.

16. Berry A, Brimacombe J, Keller C, Verghese C. Pulmonary airway resistance with the endotracheal tube versus laryngeal mask airway in paralyzed anesthetized adult patients. Anesthesiology 1999; 90: 395-7.

17. Jagannathan N, Sommers K, Sohn LE, Sawardekar A, Shah RD, Mukherji II, et al. A randomized equivalence trial comparing the i-gel and laryngeal mask airway Supreme in children. Paediatr Anaesth 2013; 23: 127-33.

18. Beringer RM, Kelly F, Cook TM, Nolan J, Hardy R, Simpson T, et al. A cohort evaluation of the paediatric i-gel $\left({ }^{\mathrm{TM}}\right)$ airway during anaesthesia in 120 children. Anaesthesia 2011; 66: 1121-6.

19. Richez B, Saltel L, Banchereau F, Torrielli R, Cros AM. A new sin- gle use supraglottic airway device with a noninflatable cuff and an esophageal vent: an observational study of the i-gel. Anesth Analg 2008; 106: 1137-9.

20. Hardman JG, Wills JS. The development of hypoxaemia during apnoea in children: a computational modelling investigation. $\mathrm{Br}$ J Anaesth 2006; 97: 564-70.

21. Derbyshire DR, Chmielewski A, Fell D, Vater M, Achola K, Smith G. Plasma catecholamine responses to tracheal intubation. Br J Anaesth 1983; 55: 855-60.

22. Eschertzhuber S, Brimacombe J, Kaufmann M, Keller C, Tiefenthaler W. Directly measured mucosal pressures produced by the i-gel ${ }^{\mathrm{TM}}$ and Laryngeal Mask Airway Supreme ${ }^{\mathrm{TM}}$ in paralysed anaesthetised patients. Anaesthesia 2012; 67: 407-10.

23. Park SK, Choi GJ, Choi YS, Ahn EJ, Kang H. Comparison of the i-gel and the laryngeal mask airway proseal during general anesthesia: a systematic review and meta-analysis. PLoS One 2015; 10: $\mathrm{e} 0119469$.

24. Beylacq L, Bordes M, Semjen F, Cros AM. The i-gel, a singleuse supraglottic airway device with a non-inflatable cuff and an esophageal vent: an observational study in children. Acta Anaesthesiol Scand 2009; 53: 376-9.

25. Kim H, Lee S, Shin HJ, Lee JH, Choi SR, Chung CJ. A clinical evaluation of i-gel ${ }^{\mathrm{TM}}$ during general anesthesia in children. Anesth Pain Med 2015; 10: 46-51. 